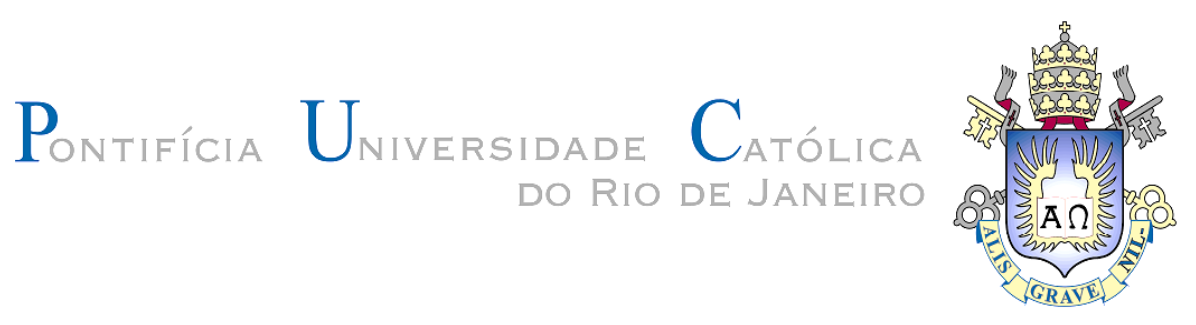

Carlos Alberto Luza Huillca

Estudo experimental do comportamento geomecânico do travertino

Dissertação apresentada como requisito parcial para obtenção do grau de Mestre pelo Programa de PósGraduação em Engenharia Civil do Departamento de Engenharia Civil da PUC-Rio.

Orientador: Prof. Eurípedes do Amaral Vargas Júnior

Rio de Janeiro

Maio de 2014 


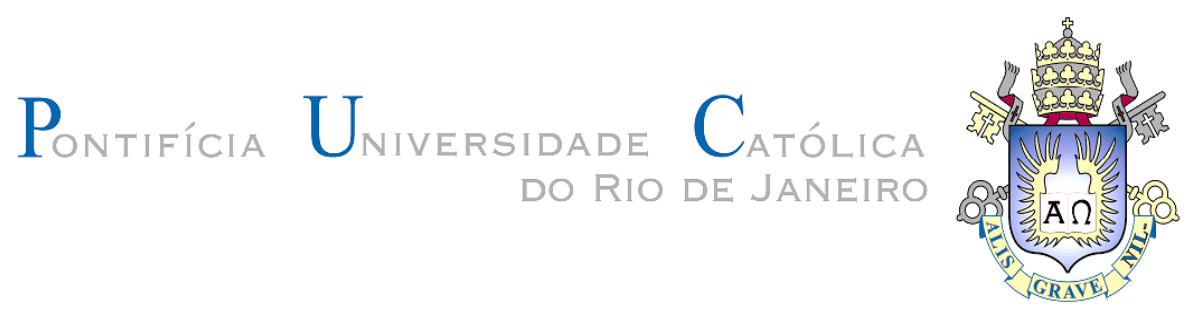

Carlos Alberto Luza Huillca

\section{Estudo experimental do comportamento geomecânico do travertino}

Dissertação apresentada como requisito parcial para obtenção do grau de Mestre pelo Programa de PósGraduação em Engenharia Civil do Departamento de Engenharia Civil do Centro Técnico Científico da PUCRio. Aprovada pela Comissão Examinadora abaixo assinada.

Prof. Eurípedes do Amaral Vargas Júnior Orientador Departamento de Engenharia Civil - PUC- Rio

Prof. Emilio Velloso Barroso Universidade Federal do Rio de Janeiro

Erick Slis Raggio Santos CENPES/Petrobras

Prof. José Eugenio Leal Coordenador Setorial do Centro

Técnico Científico - PUC-Rio

Rio de Janeiro, 23 de maio de 2014 
Todos os direitos reservados. É proibida a reprodução total ou parcial do trabalho sem autorização da universidade, do autor e do orientador.

\section{Carlos Alberto Luza Huillca}

Graduou-se em Matemática e Engenharia Geológica na UNSA (Universidade Nacional de San Agustín ArequipaPerú) em 2011. Ingressou no curso de mestrado em engenharia Civil - Geotecnia da Pontifícia Universidade Católica do Rio de Janeiro no ano 2012, desenvolvendo dissertação na linha de pesquisa de Geotecnia Experimental com enfoque na linha de Mecânica das Rochas e Geologia de Engenharia.

Ficha Catalográfica

Luza Huillca, Carlos Alberto

Estudo experimental do comportamento geomecânico do travertino / Carlos Alberto Luza Huillca; orientador: Eurípedes do Amaral Vargas Júnior. - 2014.

149 f.: il. (color.); $30 \mathrm{~cm}$

Dissertação (mestrado)-Pontifícia Universidade Católica do Rio de Janeiro, Departamento de Engenharia Civil, 2014.

Incluí referências bibliográficas.

1. Engenharia civil - Teses. 2. Travertino 3. Caracterização geomecânica. 4. Resistência. 5. Porosidade. I. Eurípedes do Amaral Vargas Júnior. II. Pontifícia Universidade Católica do Rio de Janeiro. Departamento de Engenharia Civil. III. Título.

CDD: 624 
A meus pais Serafín, Justina e irmãos pelo apoio e confiança. 


\section{Agradecimentos}

Primeiramente a Deus, por sua presença em cada dia de mi vida, e pela força e coragem concedida durante a realização deste trabalho.

A meus pais Serafín e Justina por todo o carinho, atenção e tempo dedicado na minha formação e educação, sem vocês não teria chegado aonde cheguei.

A meus Irmãos Luz Marina, Edith, María, Patricia, Julio, Cecilia e primos Biviano, Claudio, Pablito e Cipriano pelo exemplo de dedicação e entusiasmo, assim como pelo apoio durante este período de muitas renuncias.

Ao meu orientador Professor Eurípedes do Amaral Vargas Jr. pelo estimulo e parceria na realização deste trabalho.

Ao professor Franklin Antunes, pela amizade e ensinamentos pessoais e professionais transmitidos ao longo desta pesquisa.

A meus amigos da PUC-rio, em especial a Mirian Escalaya, Ronald Macazana, Francisco Cruz, Gary, Lidia, Perlita, Angela, Rosana e Fredy pelos conselhos, amizade e parceria, verdadeiramente pessoas admiráveis e inesquecíveis.

A meus caros amigos Sugey e Roberto que sempre estiveram prontos a ajudar-me desde meu primer dia no Brasil.

A todos do laboratório de Geotecnia e Estruturas da PUC-rio, em especial aos meus amigos Amaury e Euclides.

Ao pessoal do laboratório de mecânica de rochas e de tomografias de raios $-\mathrm{X}$ do CENPES/PETROBRAS, em especial a Flavia, Bete, Erick Slis, Francisco Ferreira, Marcos Soares, Rafael, Igor pela contribuição na realização dos ensaios de microtomografia e as orientações e sugestões na realização dos ensaios uniaxiais e triaxiais.

Ao Dario sua amizade e apoio na reconstituição das imagens do micro-tomógrafo e pelo acesso ao software Mimics 16.0.

Ao Guillermo e ao pessoal do laboratório da firma Martins \& Campelo, José Campelo, Johan e Watson pelo apoio e guia durante a realização dos ensaios de 
resistência mecânica.

Ao Departamento de Geologia da UFRJ e ao pessoal que labora nesta prestigiosa universidade especialmente ao professor Emilio e a Melissa pela contribuição no ensinamento dos ensaios triaxiais, assim também aos técnicos Tarciso Abreu, Sidney e Osorio, por sua grande competência profissional na elaboração das diferentes amostras usadas nesta pesquisa.

Ao professor Alexandre do Departamento de Geologia da UERJ.

Aos professores da Engenharia Civil da PUC-Rio pelos ensinamentos transmitidos durante estes dois anos de mestrado.

Ao professor Pablo Meza e ao Dr. Fernando Zuñiga y Rivero por seu apoio e motivação na realização do mestrado.

A Rita de Cássia, por sua paciência, apoio e informação brindada durante todo o mestrado.

A CAPES e à PUC-Rio, pelos auxílios concedidos, sem os quais este trabalho não poderia ter sido realizado.

Enfim, a todos os que de certa forma contribuíram a cumprir este grande objetivo que escolhi.

Muito obrigado. 


\section{Resumo}

Luza Huillca, Carlos Alberto; Vargas Júnior, Eurípedes do Amaral (orientador). Estudo experimental do comportamento geomecânico do travertino. Rio de Janeiro, 2014. 149p. Dissertação de Mestrado Departamento de Engenharia Civil, Pontifícia Universidade Católica do Rio de Janeiro.

O Travertino é uma rocha amplamente usada no mundo como rocha ornamental, mas suas propriedades petrológicas e mecânicas análogas a rochas carbonáticas de reservatório influenciaram em sua escolha para a compreensão do comportamento geomecânico destas formações. Neste sentido, este trabalho procurou analisar o comportamento geomecânico do Travertino Romano mediante um programa experimental que incluiu uma caracterização da petrologia, da estrutura porosa e do comportamento mecânico da rocha. Foi determinada sua estrutura e textura sedimentar, composição química, mineralógica e índices físicos. Além disso, sua complexa estrutura porosa foi caracterizada por análise de imagens 2D e 3D geradas no microscópio óptico e no micro-tomógrafo de raios$\mathrm{X}$, com a determinação da presença de macro e micro poros, distribuídos aleatoriamente na rocha com uma baixa conectividade, assim mesmo foi também estabelecida uma relação entre a porosidade e a resistência. Analisaram-se também os resultados dos ensaios de resistência mecânica, que tanto para o estado de tensão uniaxial como triaxial mostraram um decréscimo da resistência com o incremento da porosidade, apresentando um comportamento frágil na maioria dos casos.

\section{Palavras-chave}

Travertino; caracterização geomecânica; resistência; porosidade. 


\section{Abstract}

Luza Huillca, Carlos Alberto; Vargas Júnior, Eurípedes do Amaral (advisor). Experimental study of the geomechanical behavior of travertine. Rio de Janeiro, 2014. 149p. MSc. Dissertation - Departamento de Engenharia Civil, Pontifícia Universidade Católica do Rio de Janeiro.

The Travertine is a rock widely used in the world as an ornamental rock, but their analogous petrological and mechanical properties to carbonate rocks of reservoir influenced his choice to be able to understand the geomechanical behavior of these formations. Thus, this study sought to analyze the geomechanical behavior of Roman Travertine through an experimental program that included a characterization of petrology, the porous structure and mechanical behavior of the rock. Sedimentary structure and texture, chemical, mineralogical composition and physical indexes was determined. Moreover, Its complex porous structure was characterized by analysis of $2 \mathrm{D}$ and $3 \mathrm{D}$ images generated in the optical microscope and X-Ray micro CT-scanner, with the determination of the presence of macro and micro pores, randomly distributed in the rock with a low connectivity, so it was also established a relationship between the porosity and strength. Also analyzed the test results of mechanical strength, both to the state of uniaxial to triaxial stress showed a decrease in resistance with increasing porosity, with a brittle behavior in most cases.

\section{Keywords}

Travertine; geomechanical characterization; strength; porosity. 


\section{Sumário}

1 Introdução 22

1.1. Objetivos 23

1.2. Organização 23

2 Rochas Carbonáticas $\quad 25$

2.1.1. Constituintes das Rochas Carbonáticas 26

2.2. Classificação das Rochas Carbonáticas 28

2.2.1. Classificação de Folk 29

2.2.2. Classificação de Riding 31

2.3. O Travertino 33

2.3.1. Classificação do Travertino 34

2.4. Porosidade e Comportamento Mecânico 36

2.4.1. Estrutura Porosa 36

2.4.2. Resistência Mecânica dos Travertinos e sua Relação com a Porosidade 41

2.4.3. Resistência Mecânica e a Deformação 44

2.5. Micro-tomografia Computadorizada de Raios-X 45

3 Material $\quad 47$

3.1. Material Utilizado $\quad 47$

3.2. Travertino Romano 47

3.3. Contexto Geológico 48

3.4. Contexto Estratigráfico 50

4 Métodos 53

4.1. Considerações Iniciais 53

4.2. Caracterização Química 53

4.2.1. Fluorescência de Raios-X (XRF) 53

4.2.2. Análise da Química Elementar (EA) 54 
4.3. Caraterização Mineralógica 55

4.4. Caracterização Petrológica 56

4.4.1. Preparação das Lâminas Delgadas 56

$\begin{array}{ll}\text { 4.4.2. Análise Petrográfico } & 57\end{array}$

4.5. Caracterização da Estrutura Porosa 58

4.5.1. Preparação dos Corpos de Prova 58

4.5.2. Análise com Microscópio Eletrônico de Varredura 60

4.5.3. Micro-tomografia Computadorizada de Raios-X 60

4.6. Determinação de Índices Físicos 62

4.7. Caracterização da Resistência Mecânica 63

4.7.1. Equipamentos 63

4.7.2. Ensaios Uniaxiais e Triaxiais 70

$\begin{array}{ll}5 \text { Resultados } & 74\end{array}$

5.1. Composição Química 74

5.2. Análise Mineralógica 76

5.3. Análise Macroscópica 76

5.4. Análise com Microscópio Óptico 77

5.5. Analise com Microscópio Eletrônico de Varredura 79

5.6. Estrutura porosa 82

5.6.1. Análise com Microscópio Óptico 82

5.6.2. Análise com Microscópio Eletrônico de Varredura 84

5.6.3. Análise com Micro-tomógrafo de Raios-X 86

5.7. Índices Físicos 94

5.8. Resistência Mecânica 98

5.8.1. Resistência Uniaxial 98

5.8.2. Resistência Triaxial 104

5.9. Correlações 111

6 Conclusões 120

6.1. Sugestões para Trabalhos Futuros 122

7 Referências Bibliográficas $\quad 124$ 
Apêndice I Imagens da Microscopia eletrônica de Varredura

Apêndice II Imagens 3D do Micro CT e Perfis da Porosidade

132

Apêndice III Corpos de Prova Fraturados

146 


\section{Lista de figuras}

Figura 2.1 Formas cristalográficas típicas da calcita e dolomita. (Fonte: Ahr, 2008)

Figura 2.2 Esparita como material intergranular (esquerda) e Micrita de cor escuro e granulometria mais fina (direita). 26

Figura 2.3 Grãos aloquímicos (fonte: Reveco, 2005; Terra et al.,2010). 27

Figura 2.4 Subdivisão da Classificação das rochas carbonáticas (Ahr, 2008) 28

Figura 2.5 Classificação gráfica de Folk 1962 (Fonte: Modificado Folk, 1962). 29

Figura 2.6 Espectro textural para sedimentos carbonáticos de Folk 1962 (Fonte:

Domingues, 2011). 30

Figura 2.7 Escala de tamanho de grãos para Rochas Carbonáticas de Folk 1962

(Fonte: Modificado Folk,1962). 31

Figura 2.8 Classificação de Riding: Diagrama triangular MSC, a) dominados pela matriz de suporte: Agglutinated Microbial Reefs, Cluster Reefs, Segment Reefs, Carbonato Mud Mounds. b) dominados pelo suporte esquelético: Frame Reefs. c) dominados pelo cimento de suporte: Cement Reefs (Fonte: Riding 2002).

Figura 2.9 Representação esquemática da Classificação das Rochas Carbonáticas de Riding (Fonte: Riding 2002). 32

Figura 2.10 Cement Reefs orgânicos e carbonatos Mud Mound plotados em um

Diagrama MSC (Fonte: Riding, 2002). 33

Figura 2.11 Classificação unificada dos travertinos, Pentecost \& Viles (1994)

(Fonte: Domingues, 2011). 34

Figura 2.12 Tufa calcária (a) e travertino laminado (b). 35

Figura 2.13 Espaços vazios entre os grãos mostrando um corpo de poro e caminhos alongados conectando os poros, que são descritos como gargantas.

Figura 2.14 Classificação da porosidade de Choquette \& Pray (1970). (Fonte:

Modificado Scholle \& Scholle, 2003). 38

Figura 2.15 Classificação genética da porosidade em rochas carbonáticas de Ahr 2008 (Fonte: Ahr, 2008). 
Figura 2.16 Resistência em função da porosidade para diversos travertinos e tufas Carbonáticas (Fonte: Garcia-del-Cura et al., (2012)).

Figura 2.17 Valores do UCS para o travertino em função do peso específico seco

(Fonte: Çobanoğlu \& Çelik, 2012).

Figura 3.1 Esquema da formação do travertino de Tivoli na bacia do Acque Albule pela descarbonização dos calcários do substrato pelas águas Hidrotermais (Fonte: Modificado Filippis et al., 2013a).

Figura 3.2 Mapa geológico de Roma, Itália central, mostrando a localização da cidade de Tivoli, e a bacia do Acque Albule, com o planalto do travertino de Tivoli (Fonte: Modificado Filippis et al., 2013a).

Figura 3.3 configuração das Placas tectônicas no cretáceo superior, mostrando os margens continentais adjacentes durante movimento NW da placa Africana: 84 Ma, o início da formação do cinturão dos Apenninos (Fonte: Handy et al., 2010).

Figura 3.4 Mapa Geológico do planalto do travertino Tivoli e seções transversais dos depósitos do travertino separadas pelas superfícies de erosão S1,S2, S3, S4, S5 (Fonte: Modificado Filippis et al., 2013b).

Figura 3.5 Esquema da sequência estratigráfica da Bacia Acque Albule com seus principais litotipos (Fonte: Modificado Gasparini et al., 2002).

Figura 4.1 Espectrômetro de Fluorescência de Raios X por Energia Dispersiva 800 HS Shimadzu da PUC- Rio.

Figura 4.2 Analisador elementar EA 1112 Thermo Eléctron, PUC - Rio. 54

Figura 4.3 Vista do interior do difratômetro D8 DISCOVER BRUKER da PUCRio.

Figura 4.4 Lâminas delgadas do travertino, direções paralela e transversal à deposição.

Figura 4.5 Lupa de mesa e microscópio óptico usados na caracterização petrológica.

Figura 4.6 Amostras de mão metalizados em ouro prontas para ser usadas no MEV. 58

Figura 4.7 Preparação dos corpos de prova cilíndricos e cúbicos. 59

Figura 4.8 Corpos de prova Cúbico e cilíndrico retificados. 59

Figura 4.9 Micro - tomógrafo V Tome x L300 da marca GE/Phoenix 
(PETROBRAS/CENPES).

Figura 4.10 (a) Saturação do corpo de prova, (b) corpo de prova sendo pesado com membrana.

Figura 4.11 célula triaxial fechada sem instrumentação no corpo de prova, mostrando os componentes do corpo principal e tampa.

Figura 4.12 Esquema da célula triaxial aberta, mostrando seus componentes principais, 1. Corpo principal da tampa com fechamento rápido, 2. Came positivo de travamento rápido, 3. Came negativo de travamento rápido, 4. Conjunto de conectores elétricos e conexões hidráulicas, 5. Resistências para o aquecimento do fluido de confinamento, 6. Corpo de prova, 7. Cap inferior com três hastes de centralização, 8. Haste de carregamento axial, 9. Corpo principal da Célula triaxial.

Figura 4.13 Pressurizadores para o carregamento axial (esquerda) e lateral (direita)

Figura 4.14 Corpo de prova instrumentado com Clip gage de deslocamento radial e axial.

Figura 4.15 Curvas de calibração dos clip gage de deslocamento axial e radial (canal-0 e canal-1) usados nos ensaios.

Figura 4.16 Unidade eletro-hidráulica (esquerda) e skid com a tampa da Célula triaxial (direita).

Figura 4.17 Tela do software e Unidade de controle e monitoração. $\quad 70$

Figura 5.1 Espectros da Fluorescência de raios-X da amostra M1, M2, M3. 75

Figura 5.2 Difratograma das amostras de travertino analisadas (calcita: Ca). 76

Figura 5.3 Porosidade Vugular, fenestral e framework com dimensões milimétricas a centimétrica, se observa a pouca interconectividade dos poros tipo vugular.

Figura 5.4 Poro mostrando a calcita espática na superfície, e Micrita de uma cor cinza escuro.

Figura 5.5 (a) Garganta com calcita espática de cristais isométricos e Micrita com uma textura microcristalina de aspecto grumoso. (b), (c) Calcita microcristalina na forma de fibras ou de aspecto plumoso. $\quad 78$

Figura 5.6 Calcita cristalizada na superfície dos poros. $\quad 79$

Figura 5.7 (a) Calcita espática formada por cristais de calcita. (b) Micrita ou 
calcita microcristalina sem grãos cimentados.

Figura 5.8 Área (1) e ponto (2) analisados no EDS 80

Figura 5.9 Resultados do EDS mostrando a distinta composição do travertino 81

Figura 5.10 Fácies massiva e bandeada presentes no travertino. 82

Figura 5.11 (a) (b) corte transversal dos poros conectados e garganta na direção da deposição, (b) porosidade intercristalina na calcita espática.

Figura 5.12 Distribuição dispersa e geometria irregular dos poros do tipo fenestral e vugular observadas pelo microscópio óptico. 83

Figura 5.13 Poros conectados por gargantas de diferentes larguras e uma ampliação da garganta de menor largura. $\quad 84$

Figura 5.14 Porosidade intercristalina formada pelos grãos de calcita. $\quad 84$

Figura 5.15 Poros tipo Intrapartícula localizados dentro dos cristais de calcita de forma irregular e sem nenhum material preenchendo os poros. 85

Figura 5.16 Elementos da estrutura porosa observada pelo MEV, poros e garganta dos poros de forma irregular e distribuição dispersa.

Figura 5.17 Porosidade fenestral vista no MEV. 86

Figura 5.18 Imagens tomográficas de um corpos de prova cúbicos e cilíndrico. 87

Figura 5.19 Perfil da porosidade alongo do corpos de prova TR1. 89

Figura 5.20 Distribuição do tamanho dos poros. $\quad 90$

Figura 5.21 Plugues NX $(54 \mathrm{~mm})$ com poros maiores aos registrados no microtomógrafo. $\quad 90$

Figura 5.22 Estrutura porosa e solido dos CP TR5 e C6, reconstituída no programa Avizo Fire 8.0. 91

Figura 5.23 Solido e estrutura porosa dos CP TR2 e C2, reconstituída no programa Mimics 16.0. 91

Figura 5.24 Imagem 3D e perfil mostrando a variação da porosidade no interior do CP TR 2.

Figura 5.25 Imagem 3D e perfil mostrando a variação da porosidade no interior do CP C 2.

Figura 5.26 Objeto solido criado no Mimics 16.0 mostrando a conectividade dos poros.

Figura 5.27 porosidade obtida na reconstituição da imagem 3D convertida a voxels. 
Figura 5.28 Relação dos valores da porosidade do processamento de imagem $\mathrm{J}$ e do Mimics.

Figura 5.29 Gráfico da massa especifica seca em função da porosidade, obtidos na determinação dos índices físicos.

Figura 5.30 Comparação dos valores da porosidade obtidos experimentalmente (porosidade efetiva) e nos programas Image J e Mimics.

Figura 5.31 Correlações entre a porosidade efetiva (experimental) e a porosidade do processamento das imagens do micro-tomógrafo.

Figura 5.32 Corpos de prova testados com (TR3) e sem (TR13) a massa corrida preenchendo os poros.

Figura 5.33 (a) Curva tensão axial versus deformação axial dos ensaios de resistência uniaxial, (b) Deformação volumétrica em função da deformação axial.

Figura 5.34 Corpo de prova TR 3 depois do ensaio de compressão uniaxial, com ruptura tipo fendilhamento acompanhada de colapso dos poros.

102

Figura 5.35 Corpo de prova TR 9 fraturado devido ao colapso de poros durante o ensaio de resistência uniaxial.

102

Figura 5.36 Classificação da rocha intacta de Deere \& Miller (1966)

103

Figura 5.37 Membrana furada e poros colapsados no corpo de prova TR 12. 104

Figura 5.38 Variação do módulo elástico com a deformação axial.

105

Figura 5.39 (a) Curvas tensão desviadora versus deformação axial, (b) Curva deformação volumétrica versus a deformação axial.

Figura 5.40 Corpos de prova TR5 e TR6 fraturados

Figura 5.41 Círculos de Mohr e envolventes de ruptura de Mohr Coulomb (verde) e Hoek \& Brown (vermelha) plotados no espaço $\tau$ vs $\sigma$ no programa RocData 3.0.

108

Figura 5.42 Envoltórias de Mohr - Coulomb e Hoek \& Brown no espaço $\sigma_{1}$ vs $\sigma_{3}$ obtidas dos ensaios triaxiais e uniaxiais.

Figura 5.43 Envoltória de ruptura de Kim \& Lade para o travertino.

Figura 5.44 Influência do confinamento e da porosidade na resistência da rocha.

Figura 5.45 Faixa da variação da envoltória de ruptura.

Figura 5.46 Influência da porosidade na resistência uniaxial 
Figura 5.47 Distribuição do tamanho dos poros para o CP TR2, com porosidade menor a $10 \%$.

Figura 5.48 Propagação da fratura no ensaio de resistência mecânica. 113

Figura 5.49 Massa especifica seca em função da porosidade. 114

Figura 5.50 Resistência uniaxial em função da massa especifica seca. 114

Figura 5.51 Gráficos do módulo de Young em função da porosidade. 115

Figura 5.52 Gráficos de correlação do módulo razão em função da porosidade. 116

Figura 5.53 Gráficos de correlação do módulo de Young em função da resistência.

116

Figura 5.54 Gráficos de correlação da Resistência uniaxial em função da porosidade de diferentes pesquisas.

Figura 5.55 Correlações da Resistência uniaxial em função da massa especifica seca de diferentes pesquisas. 


\section{Lista de tabelas}

Tabela 2.1 Comparação da porosidade dos arenitos e calcários (Rodriguez, 2006).

Tabela 2.2 Estruturas, texturas e tipos de poros mais representativos dos travertinos e tufas Garcia-del-Cura et al. (2012). 42

Tabela 4.1 Processamento das imagens de micro CT para determinar a porosidade usando o programa Image J. $\quad 61$

Tabela 4.2 Especificações técnicas dos extensómetros axial e radial. $\quad 68$

Tabela 4.3 Procedimento estabelecido para a montagem do corpo de prova e a preparação da célula triaxial para os ensaios uniaxiais e triaxiais. $\quad 71$

Tabela 4.4 Distribuição do tipo de ensaio, tensão confinante e taxa de carregamento a ser aplicado em cada um dos corpos de prova. 73

Tabela 5.1 Composição química do travertino Romano. 74

Tabela 5.2 Resultados da difração de raios-X, mineralogia do travertino. 76

Tabela 5.3 Porosidade média obtida das imagens do micro CT. 88

Tabela 5.4 Tipo de poros de acordo a seu diâmetro (Fonte: Modificado Knackstedt et al., 2006).

Tabela 5.5 Valores da porosidade determinada no programa Mimics 94

Tabela 5.6 Propriedades físicas do travertino. 95

Tabela 5.7 Resultados dos ensaios de resistência uniaxial e módulos elásticos e módulo razão 99

Tabela 5.8 Resultados dos ensaios triaxiais, parâmetros elásticos e porosidade. 104

Tabela 5.9 Parâmetros dos critérios de ruptura Mohr-Coulomb, Hoek-Brown e $\begin{array}{ll}\text { Kim-Lade. } & 108\end{array}$

Tabela 5.10 Parâmetros para os limites das envolventes de ruptura superior e inferior do travertino. 110

Tabela 5.11 Equações de regressão e índices de correlação da resistência uniaxial em função da porosidade de diferentes estudos.

Tabela 5.12 Equações de regressão e índices de correlação da resistência uniaxial em função da massa especifica seca de diferentes estudos. 


\section{Lista de Abreviaturas}

ASTM

ABNT

SAE

EA

CENPES

CP

DCMM

EDS

XRF

FE

FE BSL

$H \& B$

LACQ

Ltda.

MEV

micro CT

$\mathrm{Ma}$

M - C

NBR

PUC Rio

psi

ISRM

Conf.

UCS

UERJ

UFRJ

Volt.
American Society for Testing and Materials.

Associação Brasileira de Normas Técnicas

American Iron and Steel Institute

Analise da química elementar.

Centro de Pesquisa da Petrobras

Corpo(s) de prova.

Departamento de Ciência dos Materiais e Metalurgia.

Energy dispersive $\mathrm{x}$-ray detector

Fluorescência de Raios-X.

Fundo escala

Fundo escala baixa frequência de aplicação de pressão

Hoek \& Brown

laboratório de Laboratório de Análise e Caracterização

Química

Limitada.

Microscópio eletrônico de varredura ou microscopia

eletrônica de varredura.

Micro-tomografia computadorizada de raios- $X$

milhões de anos.

Mohr-Coulomb

Norma Brasileira

Pontifícia Universidade Católica de Rio de Janeiro.

Pounds per Square Inch

Society International of Rock Mechanics.

Tensão confinante

Uniaxial Compressive Strength

Universidade Estadual de Rio de Janeiro

Universidade Federal de Rio de Janeiro

Voltagem 


\section{Lista de Símbolos}

\begin{tabular}{|c|c|}
\hline $\mathrm{H}$ ou h & Altura \\
\hline Al & Alumínio \\
\hline$\phi$ & Ângulo de atrito interno do material \\
\hline $2 \theta$ & Ângulo de difração \\
\hline atm & Atmosfera \\
\hline $\mathrm{cm}$ & Centímetros \\
\hline$v$ & Coeficiente de Poisson \\
\hline$c$ & Coesão \\
\hline D ou d & Diâmetro \\
\hline S & Enxofre \\
\hline $\mathrm{Sr}$ & Estrôncio \\
\hline $\mathrm{Fe}$ & Ferro \\
\hline $\mathrm{GPa}$ & Gigapascal \\
\hline $\mathrm{H}$ & Hidrogênio \\
\hline kgf & Kilogramo força \\
\hline $\mathrm{kN}$ & Kilonewton \\
\hline $\mathrm{kPa}$ & Kilopascal \\
\hline kV & Kilovoltio \\
\hline$E$ & Leste \\
\hline $\mathrm{Mg}$ & Magnésio \\
\hline$\gamma_{s}$ & Massa especifica seca aparente \\
\hline$\gamma_{t e o}$ & Massa especifica teórica \\
\hline$M_{\text {sat }}$ & Massa saturada \\
\hline$M_{\text {seca }}$ & Massa seca \\
\hline MN & Meganewton \\
\hline $\mathrm{MPa}$ & Megapascal \\
\hline $\mathrm{m}$ & Metro \\
\hline$\mu \mathrm{m}$ & Micrometros. \\
\hline $\mathrm{mm}$ & Milímetro \\
\hline $\mathrm{mV}$ & Milivoltios \\
\hline
\end{tabular}




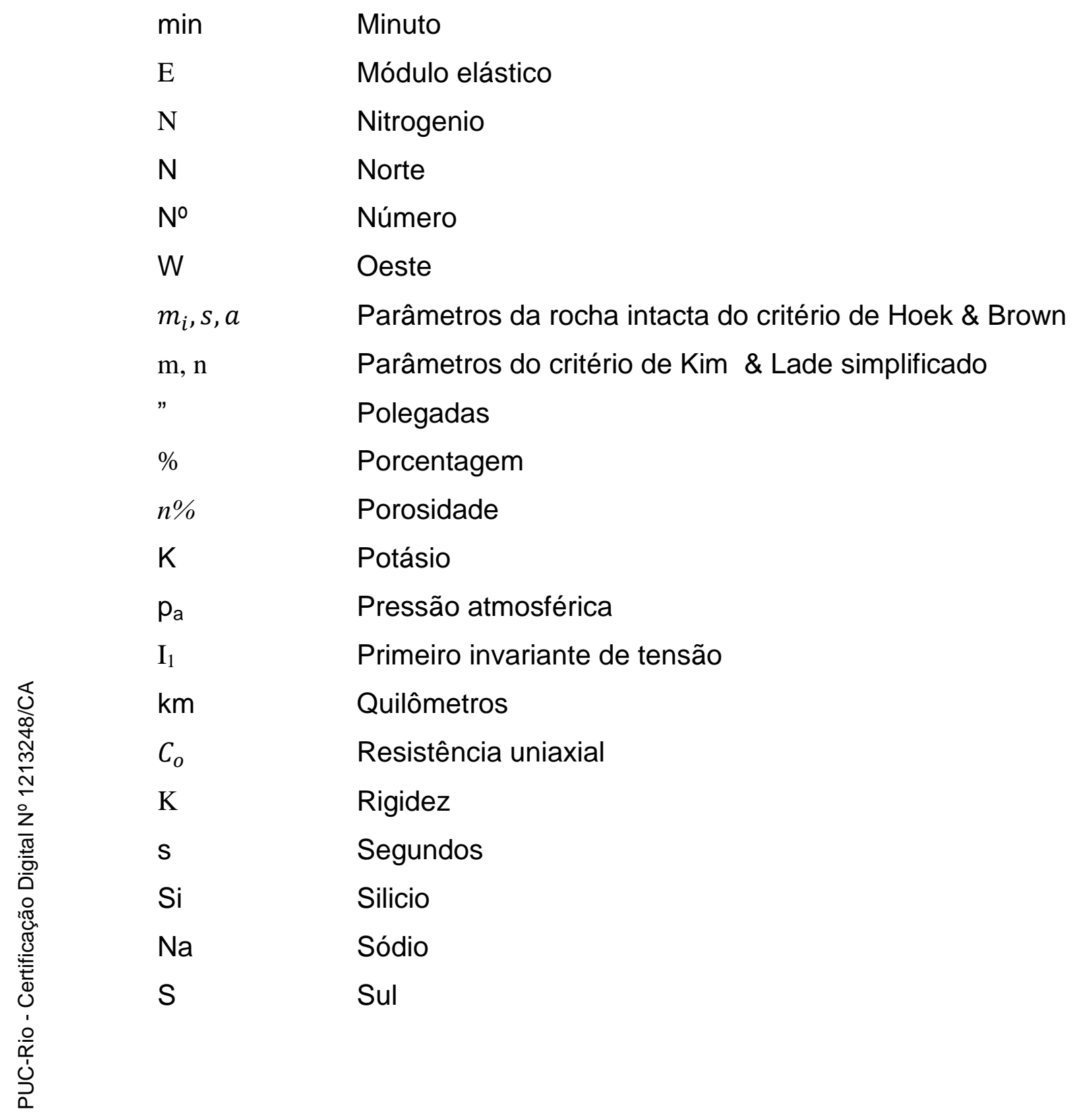

